\title{
Using Artificial Neural Networks for Meteor-Burst Communications Trail Prediction
}

\author{
Stuart Melville \\ Dep't of Computer Studies \\ ML Sultan Technikon \\ Durban, South Africa \\ stuart@wpogate.mlsultan.ac.za
}

\author{
Geoff Sutcliffe \\ Dep't of Computer Science \\ James Cook University \\ Townsville, Australia \\ geoff@cs.jcu.edu.au
}

David Fraser

Dep't of Electronic Engineering

University of Natal

Durban, South Africa

dfrase@elaine.ee.und.ac.za

The use of meteor ionisation trails as 'cheap satellites' to reflect radio waves between two points on the earth's surface is an established technique, called Meteor Burst Communications (MBC). For MBC systems to take advantage of the different amplitude and duration patterns of different trail types it is necessary to predict these patterns from features of initial signals reflected from the trails. The work described in this paper attempts to predict trail amplitude, duration, and trail type using neural networks. Results include a picture of what features of the beginning of the trail are most and least important for recognising various characteristics of the rest of the trail, some significant results as regards trail type prediction, and high correlations between actual and predicted peak amplitudes of trails. The latter is an important result.

Keywords: Neural networks, Applications to telecommunications.

\section{Introduction}

Billions of meteors ${ }^{1}$ enter the earth's atmosphere every day. There is an inverse relationship between meteor size and meteor frequency [LM+90]. Although the vast majority of the meteors are small (around the size of a grain of sand), their solar orbital velocity is high enough that on entering the upper atmosphere (between 80 and $120 \mathrm{~km}$ from the earth's surface) and burning up, they leave ionisation trails tens of kilometres long.

While Nagaoka [Nag29] was the first to postulate a connection between meteors and radio reflections, his initial hypothesis that the meteors would be impediments to radio communication was soon discovered to be incorrect. Picard [Pic31] and Skellet [Ske32] independently determined that meteors, or more specifically the trails of ionisation that they leave in their wake, could enhance radio reflection. The use of meteor ionisation trails as 'cheap satellites' to reflect radio waves between two points on the earth's surface (limited by the earth's curvature to about $2000 \mathrm{~km}$ apart) has since become an established technique. This form of communication is called Meteor-Burst Communications (MBC).

The advantages of MBC include:

- Low price. Ionised trails are free and the communication hardware is relatively cheap [Whi88, CR87].

- Robustness. The ground stations are simple and reliable [BB77, Cro77, Day82]. Meteor trails 'cannot be shot down', which makes MBC attractive for military applications [Hel87, Oet80, Boy88]. The transmission is largely impervious to electrical interference, such as polar and auroral disturbances [Hel87, DG+57].

\footnotetext{
${ }^{1}$ Strictly speaking, they are meteoroids immediately prior to entering the atmosphere, and meteors thereafter
} 
- Suitability for remote use. MBC systems have a range of up to $2000 \mathrm{~km}$, and due to their robustness and low power consumption the ground stations have low maintenance requirements [Mor88].

- Resistance to ground interception and jamming. The small footprint of the reflection means that to intercept or jam the signal requires being close to the receiving ground station [Hel87].

A number of large-scale MBC systems are in place. Important systems include:

- The United States Department of Agriculture's SnoTel telemettry system [BB77, Cro77, Day82], which comprises some 500 stations in the American West.

- The Alaska Air Command system of the US Air Force [KR86, Hof88, Sch90].

- The Chinese MBC network used by the Chinese military for communications from base stations in Beijing, Lanzhou and Urumqi to remote army camps, operating as the standard link for low priority traffic and the backup link for high priority traffic [Sch90].

A detailed review of MBC systems appears in [MF93].

The major difficulty with $\mathrm{MBC}$ is that an ionisation trail must be correctly orientated in the correct area of the sky between stations for communication to take place. The average time between usable trails varies according to known daily and seasonal cycles in meteor arrival rates, as well as being dependent on the transmitter power and the antennas used. Current state-of-the-art systems have delays of less than a second between usable trails. The channel is still sporadic however, and this means that MBC is most suited to data transmission, as opposed to real-time voice or video.

This paper first describes the different types of meteor trails, and the effect of the differnet types on the communications capacity of the MBC channel. The advantages of being able to accurately predict future trail amplitude and duration characteristics on the basis of signal reception in the early part of a trail are explained. After this various neural net approaches to the prediction of trail type, duration, and peak amplitude are discussed, and important results highlighted.

\section{The Problem}

It has long being established that there are different types of meteor trails with different duration and amplitude characteristics [Sug64, HB67, Ost85, Wei87]. Figure 1 and Figure 2 below show examples of trails recorded over an 1100km link between Arniston and Pretoria in South Africa [ML+89]. The recording starts when a signal received from the transmitter achieves a certain signal-to-noise ratio $(10 \mathrm{~dB})$, for at least $20 \mathrm{~ms}$. Thereafter samples of the signal amplitude in $\mathrm{dBm}$ are taken every $5 \mathrm{~ms}$ until the signal dips below a turn-off threshhold. (Actually the system monitors for a period of $400 \mathrm{~ms}$ after this to ensure that the turn-off has not been premature. For example, the situation where one trail with a noise spike in the middle is recorded as two distinct trails is avoided.)

Theory suggests two basic trail types, underdense and overdense [WBG84], recorded examples of these are shown in Figure 1 and Figure 2 respectively. In practise a number of other features interfere with the classic fast-rising, slow-dropping, short-duration ( $400 \mathrm{~ms}$ or so) triangle of an underdense trail, and the high-amplitude, longer-duration (seconds) smooth parabola of an overdense trail. In particular, upper atmosphere winds tend to distort these patterns considerably, as in the case in Figure 2. The TrailStar 
expert system [ML+89] distinguishes 29 distinct trail types, based on trail features (see Section 2.1 for a description of some trail features). While some of these 29 types are clearly subtypes of the two classic shapes, other cases are not clearly recognisable as being either underdense or overdense.

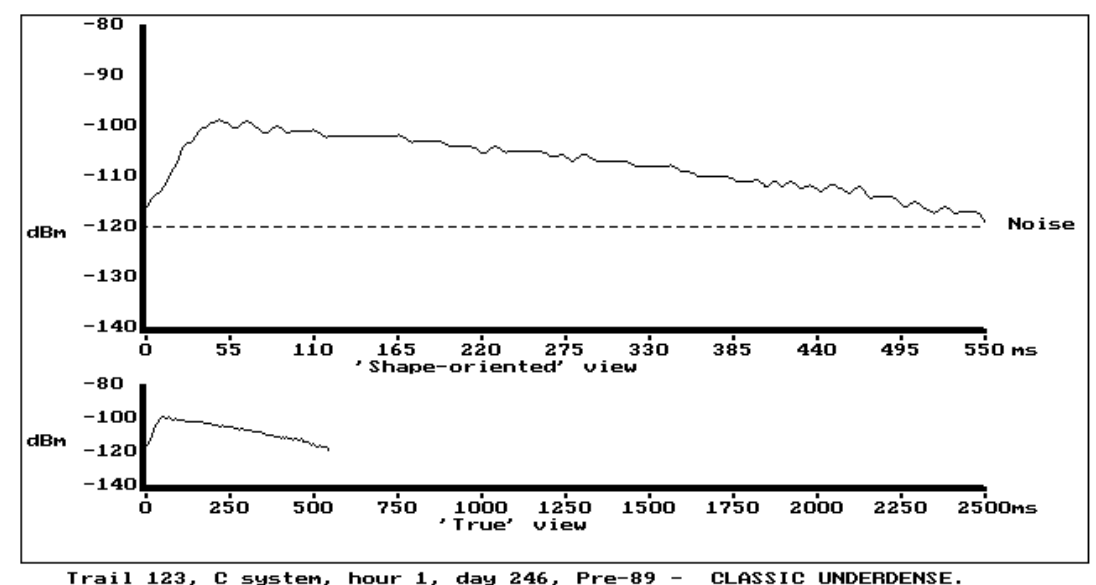

Figure 1: Underdense trail

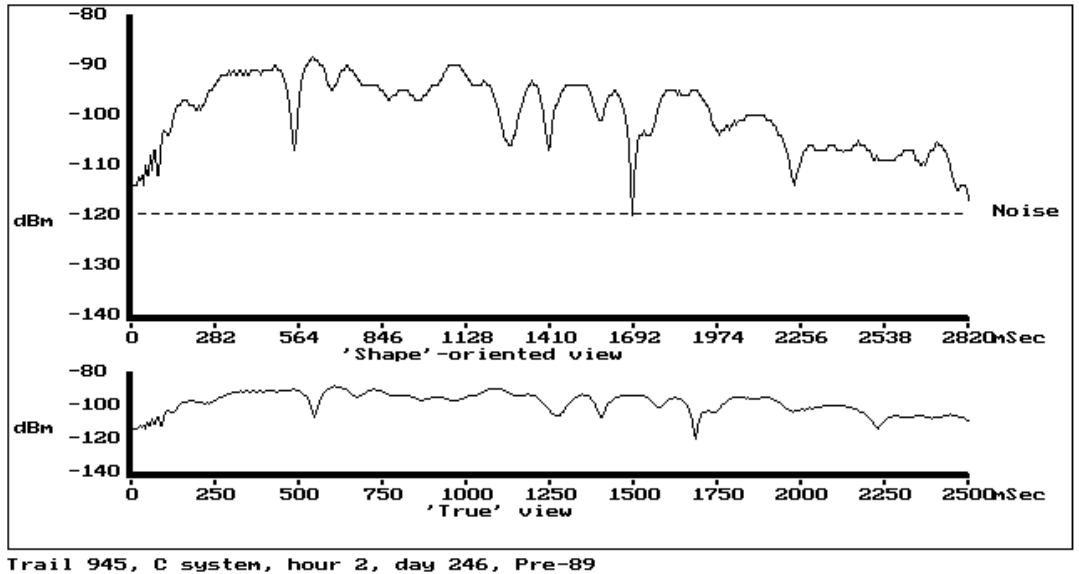

Figure 2: Overdense trail

Note on figures: Each figure shows two views of the same trail. The lower axes give a 'real' view of the trail, with one pixel plotted for each sample (samples taken every $5 \mathrm{~ms}$ ), while the upper axes give a 'shape' view, where the trail is scaled to fill the entire $\mathrm{X}$-axis. The 'shape' view is useful in studying individual trails, while the 'real' view is used for comparing trails.

The different trail shapes have major implications for MBC. Knowing the future shape of a trail would allow for more effective utilisation of channel capacity: longer duration trails provide more opportunity for data transmission, and higher-amplitude trails can support higher data rates. Importantly, the long durations and high amplitudes of the 
overdense subtypes means that they contribute the bulk of throughput, despite being the least-commonly occurring trails [LMM90]. At the same time, if wait time for short messages (which don't require a great deal of bandwidth) is at issue then MBC systems need to make optimal use of the far more commonly occurring underdense subtypes [ML92].

For communication systems to take advantage of different trail shapes it is necessary to predict the trail shape from the features of the initial part of the trail. The "initial part" of a trail is limited here to the first $50 \mathrm{~ms}$ to $100 \mathrm{~ms}$, as many entire trails in the underdense subtypes are as short as $300 \mathrm{~ms}$. The TrailStar system is inadequate for this task, as it only classifies the trails based on features of the entire trail. The TrailStar system cannot identify many of the 29 types from only this initial data, and does not consider trail amplitude and duration within types. For example, it might be known that a trail is of the sinusoidal overdense type, and thus would typically have a duration of between 2 seconds and 5 seconds, and have a peak amplitude of $15 \mathrm{dBm}$ to $20 \mathrm{dBm}$ above the background noise. However, the precise peak amplitude (and hence maximum data rate attainable) and duration (and hence usable time) would not be known, and this information could be more useful than the trail type. The work described in this paper attempts to predict trail amplitude, trail duration, and trail type as three distinct tasks, using neural networks.

\subsection{Trail Features and Types}

The TrailStar system uses over 100 features to classify trails. However, many of the features are based on trail reflection information which is not available in the first $50 \mathrm{~ms}$ to $100 \mathrm{~ms}$ of a trail. Twenty six features have been determined to be calculable from the early period of a trail together with the background noise level and the time since the previous trail was recorded. The 26 feature descriptors are:

F1: The offset of the best straight line fit.

F2: The slope of the best straight line fit.

F3: The variance of the samples from the best straight line fit.

F4: The minimum amplitude found.

F5: The maximum amplitude found.

F6: The number of samples found at the peak amplitude (possible plateaus).

F7 The position at which the peak amplitude is first encountered.

F8: The number of local minima.

F9: The number of local maxima.

F10: The number of extrema.

F11: The offset of the best straight line fit up to the peak amplitude.

F12: The slope of the best straight line fit up to the peak amplitude.

F13: The variance of the samples from the best straight line fit up to the peak amplitude.

F14: The offset of the best straight line fit from the peak amplitude onwards.

F15: The slope of the best straight line fit from the peak amplitude onwards.

F16: The variance of the samples from the best straight line fit from the peak amplitude onwards.

F17: The position where the initial amplitude rise ends.

F18: The position where the fall from the peak amplitude begins (not the same as F7 when upper plateaus at peak amplitude are encountered). 
F19: The position where the greatest $\mathrm{dBm}$ difference from the best straight line fit is found.

F20: The $\tau$ value of the slope from the peak amplitude to the end.

F21: The $x^{2}$ coefficient of the best parabola fit.

F22: The $x$ coefficient of the best parabola fit.

F23: The constant of the best parabola fit.

F24: The variance of the samples from the best parabola fit.

F25: The background noise level.

F26: The time since the previous trail.

Note that the features in this list are with respect to only the first 50 or $100 \mathrm{~ms}$ of a trail, i.e., the first 10 or 20 samples.

\section{The Neural Network Solution}

The availability of a large database of trail recordings with known type, duration and amplitude, suggested a neural network approach to predicting these values from initial trail data. For the neural network classification the 29 trail subtypes were grouped into three generic classes: the underdense class, the overdense class, and a 'non-classic' class comprising those subtypes which are not overtly underdense or overdense, or had features of both (see Figure 3 for an example). This was necessary as many of the 29 subtypes determined in $[\mathrm{ML}+89]$ are not distinguishable from the early part of the trail. In terms of the categorization done by TrailStar, the underdense class contains trail types $5,9,10,11,12,13,14,16,17,18,24$ and 29 ; the overdense class contains trail types 19, 20, 21, 26, 27, and 28; and the non-classic class contains trail types 2, 3, 4, 6, $7,8,15,22,23$ and 25 . Trail type 1 (erroneous data) is excluded from consideration.

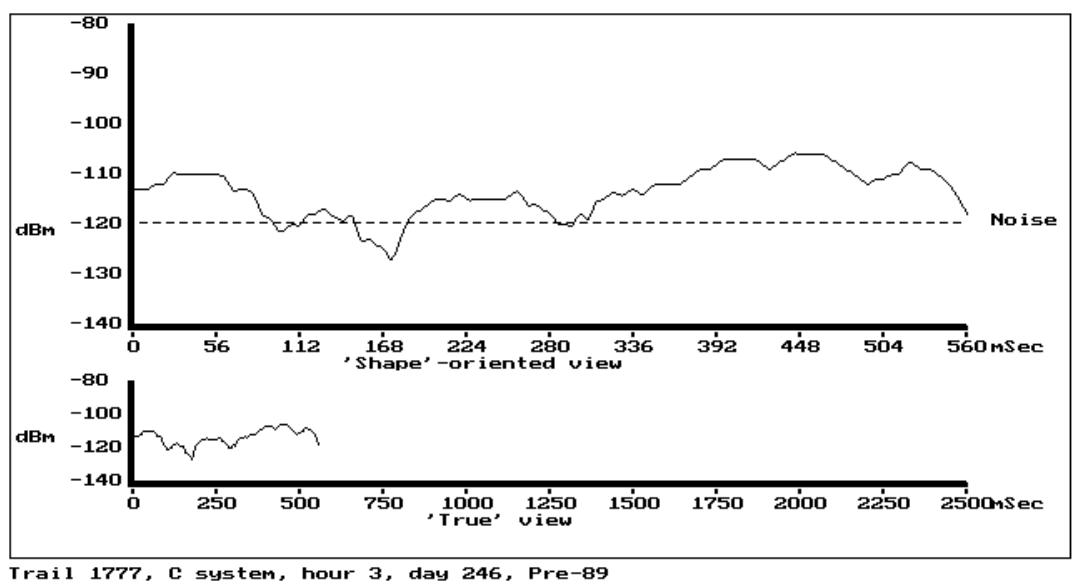

Figure 3: A sample 'non-classic' class trail

Trail recordings of 100000 trails were selected at random from the database of trail recordings. From the trail data the 26 features were calculated from the initial trail samples, as inputs to the neural network. These features were calculated for the first 20 samples $(100 \mathrm{~ms})$, and also for the first 10 samples $(50 \mathrm{~ms})$ to see if faster prediction is possible. The trails were also classified by TrailStar into one of the 29 TrailStar types 
using the entire trail data, and then put into one of the three classes. The trail class, duration, and peak amplitude formed the expected output for neural network training.

The input/output combinations were divided into two groups; a training group of 96000 examples, and a test group of just over 4000 examples. Supervised learning was then used to train the network to predict trail class, duration, and amplitude, from the 26 trail features. Neuralware's Neuralworks version 2.0 [Neu93] was used to build the required neural networks.

\subsection{Trail Type Nets and Results}

Trail classification proved to be a particularly vexing problem. Initially a backpropagation network was used with 26 input Processing Elements (one for each input feature descriptor), 58 PEs in a hidden layer, and one output PE (the trail class). Training this network gave either convergence without generalisation (low RMS error but low correlation of actual/predicted too) or did not converge at all. The next attempt was to use an LVQ network, as LVQ networks are supposedly well suited to classification type problems [Neu93]. In this network three output fields were used, allowing three different 'bit patterns' $(000,010,111)$ to represent the three trail classes. This approach also failed, with the network converging on the most common trail types, non-classic and underdense.

Neither the backpropagation nor the LVQ network was predicting overdense trails at all, and it seemed likely that this was due to the relatively low number of overdense trails in the training data (as one would expect; they only form a small proportion of trails). To counter this perceived swamping of the overdense trails, 5000 trails of each of the three classes were extracted from the 96000 , based on the fact that there were just over 5000 overdense trails available. This formed a new training set of 15000 examples with equal representation of each trail class. The 4000 test trails were left unaltered. While this step did lead to some predictions of overdense trails, correlations were still poor. Guessing that this might be due to the network being overexposed to 'happenstance' features (and so failing), a subset of the input features was used. The features were selected according to their relative importance in the TrailStar typing scheme. The subset contained just nine features, these being F2, F3, F4, F5, F7, F10, F22, F23, and F24. This improved performance somewhat, giving predicted/actual correlations of approximately 0.4 . It was felt that better could be achieved, and at this stage it was decided to add an additional hidden layer to the network to aid in the recognition of more complex patterns. This layer consisted of five PEs, making the network configuration 9 input layer PEs, 58 first hidden layer PEs, 5 second hidden layer PEs, and 1 output layer PE. It is from this configuration that the final results were obtained.

The eventual weights on the inputs (for the case where the features were derived from 20 trail samples) were as follows : 


$$
\begin{array}{lrl}
\text { F2: } & 0.259 & \\
\text { F3: } & -0.946 & \text { (The variance of the samples from the best straight line fit) } \\
\text { F4: } & -0.268 & \\
\text { F5: } & -0.261 & \\
\text { F7: } & 1.000 & \text { (The position at which the peak amplitude is first encountered) } \\
\text { F10: } & -0.667 & \\
\text { F22: } & 0.048 & \text { (The } x \text { coefficient of the best parabola fit) } \\
\text { F23: } & -0.523 \\
\text { F24: } & -0.975 & \text { (The variance of the samples from the best parabola fit) }
\end{array}
$$

\begin{tabular}{|c|c|c|c|c|c|}
\hline Trail class & \multicolumn{5}{|c|}{ Predictions } \\
\hline & Total & $\mathrm{Ud}$ & Od & Non-C & Correct \\
\hline Underdense & 1942 & 881 & 76 & 985 & $45 \%$ \\
\hline Overdense & 286 & 11 & 109 & 166 & $38 \%$ \\
\hline Non-classic & 1791 & 188 & 471 & 1132 & $63 \%$ \\
\hline Total & 4019 & 1080 & 656 & 2283 & $53 \%$ \\
\hline
\end{tabular}

\begin{tabular}{|c|c|c|c|c|c|}
\hline Trail class & \multicolumn{5}{|c|}{ Predictions } \\
\hline & Total & $\mathrm{Ud}$ & $\mathrm{Od}$ & Non-C & Correct \\
\hline Underdense & 1966 & 701 & 83 & 1182 & $36 \%$ \\
\hline Overdense & 278 & 12 & 103 & 163 & $37 \%$ \\
\hline Non-classic & 1776 & 129 & 558 & 1089 & $61 \%$ \\
\hline Total & 4020 & 842 & 744 & 2434 & $47 \%$ \\
\hline
\end{tabular}

Only the $x$ coefficient of the best parabola fit seemed to have negligible effect (weighted less than 0.1). It is interesting that the parabola and line variance measures had high weights (greater than 0.9 in magnitude) after training; such measures seem to be important descriptors. The strong role played by the peak amplitude position (weight of 1) was less expected and constitutes an important finding.

Table 1: Results of class prediction; 20 samples

Table 2: Results of class prediction; 10 samples

It is clear from the data in Table 1 and Table 2 that the network is fairly proficient at distinguishing between underdense and overdense trails, with only a very small number of underdense trails being predicted as overdense, and vice versa. The problem remaining is that the network easily confuses underdense and overdense with nonclassic. Of course this should be expected, as the non-classic class contains precisely those trails that have both overdense and underdense features.

The fact that around $50 \%$ of predictions are correct, together with the above described underdense/overdense discrimination, is particularly encouraging. The correlations look worse due to the effect of the non-classic group. Notwithstanding that, the correlations of 0.534 (prediction based on 20 samples) and 0.428 (prediction based on 10 samples) are encouraging.

\subsection{Duration Nets and Results}

A good prediction of trail duration is highly unlikely. Some types of trails, such as rectified sines (see Figure 4), repeat a basic pattern $n$ times. There is no way to detect, 
on the basis of the first $50 \mathrm{~ms}$ or $100 \mathrm{~ms}$, just what $n$ is going to be. However an attempt at neural network prediction was made.

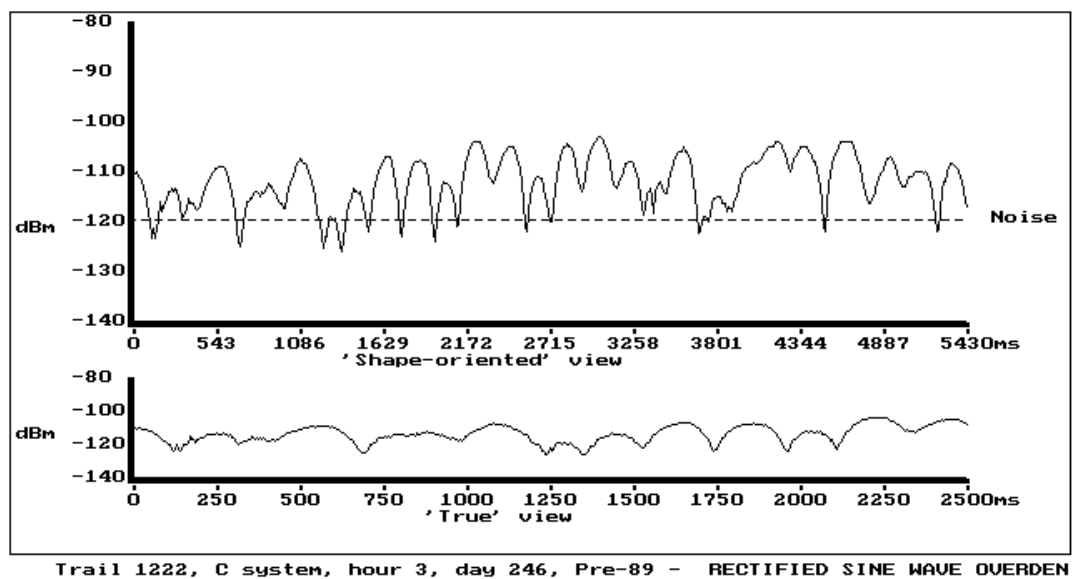

Figure 4: Rectified sine trail

The backpropagation network used here had 26 input PEs, 58 PEs in a hidden layer, and one output PE (the duration). After extensive training (several million epochs) no further convergence seemed to be occurring, and the weights were fairly evenly distributed amongst inputs, indicating that most of the inputs were having a significant effect on the network. Input weights were as follows after training:

$\begin{array}{llllll}\text { F1: }-0.470 & \text { F2: } 0.585 & \text { F3: }-0.948 & \text { F4: }-0.244 & \text { F5: } 0.167 \\ \text { F6: }-0.579 & \text { F7: } 0.789 & \text { F8: }-0.677 & \text { F9: }-0.818 & \text { F10: }-0.712 \\ \text { F11:-0.712 } & \text { F12: } 0.813 & \text { F13:-0.998 } & \text { F14: }-0.239 & \text { F15: }-0.856 \\ \text { F16: }-0.952 & \text { F17: } 0.789 & \text { F18: } 0.789 & \text { F19: } 0.867 & \text { F20: } 0.552 \\ \text { F21: } 0.052 & \text { F22: } 0.138 & \text { F23:-0.517 } & \text { F24: }-0.934 & \text { F25: }-0.999 \\ \text { F26: } 0.000 & & & & & \end{array}$

(Values shown are for the 20 sample case, the 10 sample results are similar.)

The features with weights of particular interest are:

F3: -0.948 (The variance of the samples from the best straight line fit)

F13:-0.998 (Variance from the best straight line fit up to the peak amplitude)

F16:-0.952 (The variance from the best straight line fit from the peak amplitude onwards)

F21: 0.052 (The $\mathrm{x} 2$ coefficient of the best parabola fit)

F24: -0.934 (The variance of the samples from the best parabola fit)

F25: -0.999 (The background noise level)

F26: 0.000 (The time since the previous trail was encountered)

After the classification results it came as no surprise that the variance measures were of great importance (weighted greater than 0.9 in magnitude). The high weight assigned to background noise is somewhat puzzling, and merits further investigation. As regards low (less than 0.1 in magnitude) weights only F21 and F26 seem to have minimal effect. 
Scatter graphs of the actual vs predicted trail durations for the 20 sample case are shown in Figure 5 below. The right-hand graph shows the more common duration trails (up to $1000 \mathrm{~ms}$ ) at higher resolution.
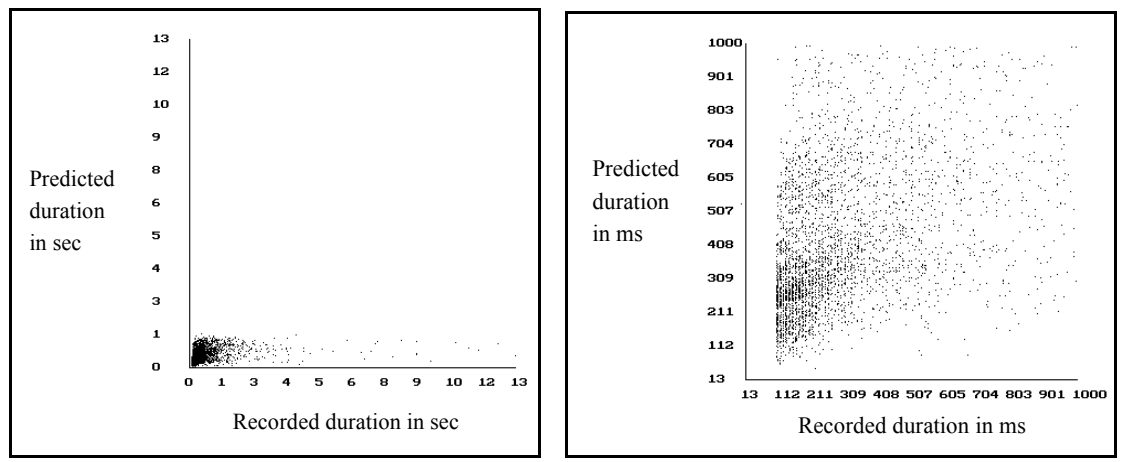

Figure 5: Duration prediction, 20 samples

It is apparent that the prediction is not particularly accurate, as was expected. The Pearson coefficients of correlation are 0.303 for the 20 sample case and 0.206 for the 10 sample case. Although these coefficients are statistically significant due to the large sample size, they indicate only a weak positive correlation between actual and predicted results. Such results are better than nothing, but work on finding more effective discriminators must continue.

\subsection{Amplitude Nets and Results}

For the amplitude prediction a backpropagation network using 26 input PEs, a hidden layer of 80 PEs, and a single output PE, was used. The network was trained on the 96000 training examples for both the 10 and 20 sample cases. Input weights were as follows after training:

$\begin{array}{llllll}\text { F1: }-0.182 & \text { F2: } 0.308 & \text { F3: }-0.887 & \text { F4: }-0.111 & \text { F5: } 0.042 \\ \text { F6: }-0.263 & \text { F7: } 0.263 & \text { F8: }-0.667 & \text { F9: }-1.000 & \text { F10: }-0.818 \\ \text { F11: }-0.744 & \text { F12: } 0.813 & \text { F13:-0.998 } & \text { F14: }-0.118 & \text { F15: }-0.847 \\ \text { F16: }-0.998 & \text { F17: } 0.789 & \text { F18: }-0.158 & \text { F19: } 0.867 & \text { F20: } 0.552 \\ \text { F21: }-0.292 & \text { F22: } 0.370 & \text { F23:-0.470 } & \text { F24: }-0.990 & \text { F25: }-0.999 \\ \text { F26: } 0.000 & & & & & \end{array}$

(Values shown are for the 20 sample case, the 10 sample results are similar)

The features with weights of particular interest are:

F3: -0.887 (The variance of the samples from the best straight line fit)

F5: 0.042 (The maximum amplitude found)

F9: -1.000 (The number of local maxima)

F13:-0.998 (Variance from the best straight line fit up to the peak amplitude)

F16:-0.998 (The variance from the best straight line fit from the peak amplitude onwards)

F24: -0.990 (The variance of the samples from the best parabola fit)

F25:-0.999 (The background noise level)

F26: 0.000 (The time since the previous trail was encountered) 
The above makes fascinating reading. It is particularly startling that the peak amplitude found in the early part of a trail seems to be of virtually no significance in predicting the peak amplitude of the entire trail. This merits further investigation. The only other feature that has little effect (weighted less than 0.1 in magnitude) is the wait time since the previous trail, a result in keeping with the duration findings.

As regards important (weight greater than 0.9 in magnitude) features, once again the variances stand out, with F13, F16 and F26 all exceeding 0.9 in absolute value. F3 is only slightly less important (magnitude of 0.887 ). Background noise again has a high weight, in keeping with the duration results, and this deserves further study. Of particular interest is that the number of local maxima found has the greatest magnitude weight of any input (1.0 in magnitude). A more detailed analysis of this important result is necessary.

Results from these networks were most impressive, as can be seen from Figure 6 below, which shows the 10 sample case. The correlation is even stronger in the 20 sample case. (The vertical striation in the Figure is because amplitude is measured discretely).

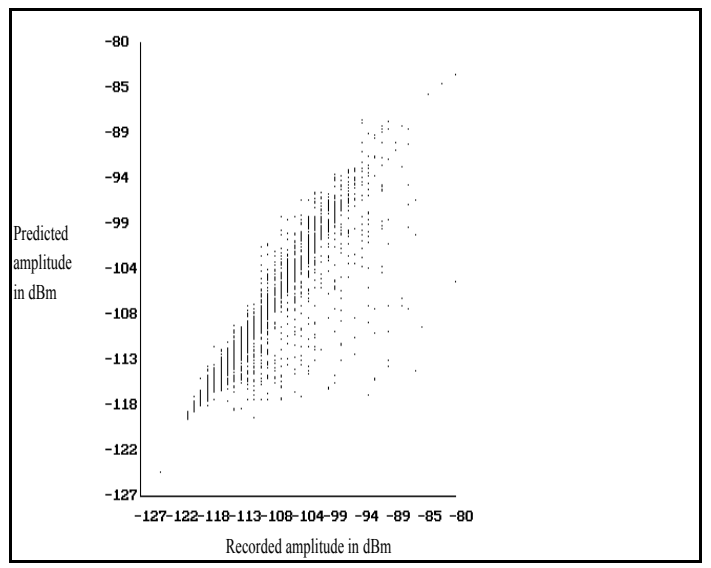

Figure 6: Amplitude prediction, 10 samples

The correlation coefficients produced were 0.911 for the 20 sample case, and 0.823 for the 10 sample case. These are high positive correlations. Having such a reliable predictor of the eventual peak amplitude from sampling just the first 50ms of the trail has tremendous and obvious implications for vast improvements in MBC throughput.

\section{Conclusion}

This paper has describes an emperical study into the use of neural nets to predict future characteristics of meteor trails. Being able to predict in real-time what communication capacity the upcoming trail will have, enables greater utilisation of the trail than is currently possible. Results include:

- A most necessary (for future work) picture of what features at the beginning of the trail are most and least important for recognising various characteristics of the rest of the trail.

- $\quad$ Some expected, though still disappointing, poor correlations between predicted and actual durations. 
- Some significant results as regards trail type prediction.

- High correlations between actual and predicted peak amplitudes of trails, even on the basis of data from just $50 \mathrm{~ms}$ at the beginning of trails. This is an important result with immediate implications for the design of MBC systems. This result justifies the entire research effort.

MBC systems have the potential to provide cheap and reliable communications in remote and developing areas. This is particularly relevant to the Pacific Rim, where large distances and inaccesiblity make the cost of installing traditional communication systems prohibitive. The Chinese MBC system [Sch90] is a working example of this. The improved performance that can be realised from real-time prediction of trail patterns will considerably enhance such systems.

Acknowledgement: This research was financed by Salbu (Pty) Ltd.

\section{References}

[BB77] Barton, M., Burke, M., SNOTEL - An Operational Data Acquisition System using Meteor-Burst Technology, paper presented at the Western Show Conference, (1977).

[Boy88] Boyle, D., Long Distance Communications - Back to Ionization, International Defence Review 21, 491-493, (1988).

[Cro77] Crook, A.G., SNOTEL : Monitoring Climatic Factors to Predice Water Supplies, Journal of Soil and Water Conservation 32, 294-295, (1977).

[CR87] Cannon, P.S., and Reed, A.P.C., The Evolution of Meteor-Burst Communication Systems, Journal of the Institution of Electronic and Radio Engineers 57, 101-112, (1987).

[Day82] Day, W.E., Meteor-Burst Communications Bounce Signals Between Remote Sites, Electron 55, 71-75, (1982).

[DG+57] Davis, G.W.L., Gladys, S.J., Lang, G.R., Luke, L.M., Taylor, M.K., The Canadian JANET System, Proceedings of the IRE 45, 1666-1678, (1957).

[HB67] Hawkins, G.S., and Brown, J.C., A Comprehensive Study of the Characteristics of Meteor Echoes - I, Smithsonian Astrophysical Observatory, Report \# NASA-CR-92667 (1967).

[Hel87] Hellweg, G.A., Meteor-Burst Communications : Is This What the Navy Needs?, Master's Thesis, Naval Postgraduate School, Monterey, U.S.A., (1987).

[Hof88] Hoff, J. A., The Utility of Meteor Burst Communications, Conference Record of the 1988 IEEE Conference on Military Communications, (1988).

[KR86] Kokjer, K.J., Roberts, T.D., Networked Meteor-Burst Data Communications, IEEE Communications Magazine 24, 23-29, (1986).

[LMM90] Larsen, J.D., Melville, S.W., and Mawrey,R.S.M., Adaptive Data Rate Capacity of Meteor-Burst Communications, Conference Record of the 1990 IEEE Conference on Military Communications, Vol 2, 40.1.1 - 40.1.5, (1990).

[LM+90] Larsen, J.D., Melville, S.W., Mawrey, R.S., Letschert, R.Y., and Goddard, W.D. Throughput Capacity of Meteor Burst Communications, Transactions of the SAIEE 81 (1), 20 - 30, (1990).

[Mor88] Morgan, E.J., Meteor Burst Communications : An Update, Signal 42, 55-61, (1988). 
[MF93] Melville, S.W., and Fraser, D.D., Meteor-burst Communication: A Review, Transactions of the SAIEE 84 (2), 60 - 68, (1993).

$[\mathrm{ML}+89]$ Melville, S.W., Larsen, J.D., Letschert, R.Y., and Goddard, W.D., The Classification of Meteor Trail Reflections by a Rule-Based System, Transactions of the SAIEE 80 (1), 104 - 116, (1989).

[ML92] Melville, S.W. and Larsen, J.D., Wait Time in Meteor-Burst Communications, Transactions of the SAIEE 83 (1), 32 - 37, (1992).

[Nag29] Nagaoka, H., Possibility of Disturbance of Radio Transmission by Meteor Showers, Proceedings of the Imperial Academy of Tokyo 5, p 632, (1929).

[Neu93] Neuralware, Inc. Neural Computing. Technical Publications Group: Pittsburgh, (1993).

[Oet80] Oetting, J.D., An Analysis of Meteor-Burst Communications for Military Applications, IEEE Transactions on Communications 38, 1591 - 1601, (1980).

[Ost85] Ostergaard, J.C., Characteristics of High-Latitude Meteor Scatter Propagation Parameters over the 45 to $104 \mathrm{Mhz}$ Band, AGARD Conference Proceedings 382, (1985).

[Pic31] Pickard, G.W., A Note on the Relation of Meteor Showers and Radio Reception, Proceedings of the IRE 19,1166-1170, (1931).

[Sch90] Schanker, J. Z., Meteor Burst Communications. Artech House, Inc., Norwood, MA, (1990).

[Ske32] Skellet, A.M., The Ionizing Effect of Meteors in Relation to Radio Propagation, Proceedings of the IRE 20, 1933-1940, (1932).

[Sug64] Sugar, G.R., Radio Propagation by Reflection from Meteor Trails, Proceedings of the IEEE 52, pp 11 - 136, (1964).

[WBG84] Weitzen, J.A., Birkemeier, W.P., and Grossi, M.D., An Estimate of the Capacity of the Meteor-Burst Channel, IEEE Transactions on Communications 32, 972-974, (1984).

[Wei87] Weitzen, J.A., A Data Base Approach to the Analysis of Meteor Burst Data, Radio Science 22, 133 - 140, (1987).

[Whi88] Whittaker, C.J., Meteor Burst Communication, Journal of Research of the Signals Institute 18, 185-190, (1988). 